\title{
Cardioprotective effect of remote preconditioning of trauma and remote ischemia preconditioning in a rat model of myocardial ischemia/reperfusion injury
}

\author{
QING CHAI ${ }^{1}$, JIN LIU $^{1}$ and YANG HU ${ }^{2}$ \\ Departments of ${ }^{1}$ Critical Medicine and Anesthesiology and ${ }^{2}$ Thoracic and Cardiovascular Surgery, \\ West China Hospital, Sichuan University, Chengdu, Sichuan 610041, P.R. China
}

Received June 7, 2014; Accepted February 5, 2015

DOI: $10.3892 / \mathrm{etm} .2015 .2320$

\begin{abstract}
Remote ischemia preconditioning (RIPC) and remote preconditioning of trauma (RPCT) are two methods used to induce a cardioprotective function against ischemia/reperfusion injury (IRI). However, the underlying mechanisms of these two methods differ. The aim of the present study was to investigate the cardioprotective function of the two methods, and also observe whether combining RIPC with RPCT enhanced the protective effect. In total, 70 male Sprague Dawley rats were randomly divided into five groups, which included the sham, control, RIPC + RPCT, RPCT and RIPC groups. With the exception of the sham group, all the rats were subjected to myocardial IRI through the application of $30 \mathrm{~min}$ occlusion of the left coronary artery and $180 \mathrm{~min}$ reperfusion. Serum cardiac troponin I (cTnI) levels, myocardial infarct size (IS) and the cardiomyocyte apoptotic index (AI) were assessed. The levels of serum cTnI were lower in the experimental groups when compared with the control group (control, 58.59 $\pm 12.50 \mathrm{pg} / \mathrm{ml}$; RIPC + RPCT, 46.05 $\pm 8.62 \mathrm{pg} / \mathrm{ml}$; RPCT, $45.98 \pm 11.24 \mathrm{pg} / \mathrm{ml}$; RIPC, $43.46 \pm 5.05 \mathrm{pg} / \mathrm{ml} ; \mathrm{P}<0.05$, vs. control), and similar results were observed for the myocardial IS (control, 48.34 $\pm 6.79 \%$; RIPC + RPCT, 29.64 $4.51 \%$; RPCT, $29.05 \pm 8.51 \%$; RIPC, $27.72 \pm 6.27 \%$; $\mathrm{P}<0.05$, vs. control) and the AI (control, 31.75 $\pm 10.65 \%$; RIPC + RPCT, 18.32 $\pm 9.30 \%$;

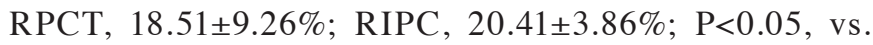
control). However, no statistically significant differences were observed among the three experimental groups $(\mathrm{P}>0.05)$. Therefore, RIPC and RPCT exhibit cardioprotective effects when used alone or in combination. However, a combination
\end{abstract}

Correspondence to: Mr. Jin Liu, Department of Critical Medicine and Anesthesiology, West China Hospital, Sichuan University, 37 Guo Xue Xiang Road, Chengdu, Sichuan 610041, P.R. China E-mail: liujinscu@sina.com

Key words: ischemia/reperfusion injury, ischemia preconditioning, remote ischemia preconditioning, remote preconditioning of trauma, myocardioprotection of RIPC and RPCT does not enhance the cardioprotective effect observed with the application of either single method. Therefore, for patients undergoing major abdominal surgery, RIPC was considered to be unnecessary, while for patients undergoing other types of non-cardiac major surgery and minimally invasive interventional surgery, RIPC may be useful. In addition, patients with embolism diseases are also liable to IRI when reperfusion treatment such as thrombolysis is conducted. Thus RIPC may also be beneficial for these patients.

\section{Introduction}

Ischemia/reperfusion injury (IRI) occurs in the myocardium and is a negative factor for the morbidity and mortality rates of inpatients, particularly for patients undergoing cardiac coronary surgery or elderly patients undergoing major surgery (1). In 1986, Murry et al (2) established the technique of ischemia preconditioning (IPC), in which periods of IRI were applied to confer an intrinsic protective mechanism that in turn decreased the extent of IRI. Subsequently in 1993, Przyklenk et al (3) found that short periods of ischemia in a distant organ were able to induce protection to target organs against sustained IRI. The authors referred to this process as remote IPC (RIPC). Several studies have demonstrated that IPC is a complicated multi-mechanism process that involves humoral and neural pathways $(4,5)$.

In 2004, Ren et al (6) identified a novel cardioprotective phenomenon, where the myocardium infarct size (IS), following IRI, was decreased by a transverse abdominal incision. The term 'remote preconditioning of trauma' (RPCT) was devised for this non-ischemic preconditioning phenomenon. In subsequent experiments, scientists have proposed that nociceptive stimulation of peripheral sensory nerves leads to the activation of the cardiac sympathetic nervous system via spinal nerves (7).

The underlying mechanisms of RIPC and RPCT are different. However, whether RIPC or RPCT exerts a stronger cardioprotective function is yet to be elucidated. In addition, whether a combination of RIPC with RPCT is able to enhance the cardioprotective function is also unclear. Thus, the present study utilized an in vivo rat model of myocardial IRI to testify these questions. 


\section{Materials and methods}

The study design was approved by the Institutional Animal Care and Use Committee of Sichuan University (Chengdu, China). All the experimental procedures were undertaken at the West China Clinical Skills Training Center and the West China School of Medicine/West China Hospital of Sichuan University.

Animal preparation. In total, 70 male Sprague Dawley rats (age, 7 weeks; weight, 250-300 g) were provided by Sichuan Provincial Laboratory Animal Public Service Center (Chengdu, China). The animals were fed the same diet and were provided with water ad libitum. The housing environment was maintained at $23^{\circ} \mathrm{C}$ and $60 \%$ humidity, with a 12 -h day and night cycle. The rats were randomly divided into five groups according to a random number table generated by a computer. The animals were anesthetized by an intraperitoneal injection of $2 \%$ sodium pentobarbitone $(50 \mathrm{mg} / \mathrm{kg}$; Sigma-Aldrich, St. Louis, MO, USA), and anesthesia was maintained with repeated administration of sodium pentobarbitone $(25 \mathrm{mg} / \mathrm{kg})$ every $60 \mathrm{~min}$. The body temperature of the rats was maintained at $37^{\circ} \mathrm{C}$ using a heating blanket (Shanghai Yuyan Instruments Co., Ltd., Shanghai, China). A tracheotomy was conducted with a $14-\mathrm{G}$ catheter (Terumo Corporation, Tokyo, Japan) for controlled ventilation, comprising a respiratory rate of 60-70 breaths/min with $40 \%$ oxygen support, $2.5-3.5 \mathrm{ml}$ tidal volume and a 1:2 inspiration/expiration ratio. A standard limb lead II electrocardiogram (Chengdu Taimeng Software Co., Ltd., Chengdu, China) was used to monitor the heart rate (HR) and ST-segment changes via subcutaneous needle electrodes. Furthermore, a 20-G catheter (Terumo Corporation, Tokyo, Japan), filled with normal saline containing heparin, was inserted into the right carotid artery to monitor the mean arterial blood pressure (MABP). HR and MABP measurements were recorded using a computer-based monitoring system (Chengdu Taimeng Software Co., Ltd).

Surgical procedure. An anterolateral thoracotomy was conducted at the left fourth intercostal space, which was subsequently accompanied by a pericardiotomy to expose the heart. The left anterior descending (LAD) branch of the left coronary artery was ligated with a 6/0 silk suture (Shanghai Pudong Jinhuan Medical Products Co., Ltd., Shanghai, China) at the midpoint between the base and apex. Changes in the ST segment, emerging arrhythmia for a short duration, regional color change at the distal ligation site and reduced MABP were useful indicators for confirming the successful establishment of myocardial ischemia. Rats were subjected to $30 \mathrm{~min}$ regional ischemia followed by $180 \mathrm{~min}$ reperfusion to establish the myocardium IRI model. Rats with hypotension (MABP of $<60 \mathrm{mmHg}$ ) that did not return to normal, or hemoglobin levels of $<50 \mathrm{~g} / \mathrm{l}$ at the end of the experiment were excluded from the study.

Experimental protocols. Animals were divided into five groups (Fig. 1). The sham group underwent a thoracotomy only, while the control group were subjected to myocardial IRI only. The RIPC + RPCT treatment group received an abdomen incision, followed by three cycles of 5 min bilateral femoral artery occlusion and reperfusion, after which the incision was sutured prior to the conduction of myocardial IRI. The RPCT group underwent an abdomen incision, which was sutured 30 min later prior to subjection to myocardial IRI. Finally, the RIPC group received three cycles of 5 min bilateral femoral artery occlusion and reperfusion, without abdomen incision, prior to the induction of myocardial IRI.

Enzyme-linked immunosorbent assays (ELISA). Blood samples were collected at the end of the experiment. Serum levels of cardiac troponin I (cTnI) were analyzed using an ELISA kit (USCN Life Science, Inc., Wuhan, China), and the data were measured on a microplate reader (Bio-Tek Instruments, Inc., Winooski, VT, USA).

Cardiac IS determination. At the endpoint of reperfusion, the LAD artery was ligated once more in order to identify the area at risk (AAR). Evan's blue dye (2\%,2 ml; Sigma-Aldrich) was injected through the right carotid artery. The rats were died from acute blood loss caused by the injection and their hearts were obtained and frozen. The heart samples were cut into $1-\mathrm{mm}$ transverse sections from the apex to the occlusion site, and the slices were immersed in 1\% 2,3,5-triphenyltertrazolium chloride (Sigma-Aldrich) phosphate buffer for $30 \mathrm{~min}$ (the buffer was adjusted to $\mathrm{pH} 7.4$ at $37^{\circ} \mathrm{C}$ ). Next, the specimens were fixed with $4 \%$ paraformaldehyde for $24 \mathrm{~h}$ to enhance the contrast of the stain. Every stained slice was scanned into a computer (Shanghai Microtek Trade Co., Ltd., Shanghai, China), and the infarct area and AAR were calculated using Image J 1.44p software (National Institutes of Health, Bethseda, MD, USA). The IS was expressed as a percentage of the infarct area over the AAR.

Apoptotic index (AI) measurements. A terminal deoxynucleotidyl transferase-mediated dUTP nick-end labeling assay (TUNEL; Promega Corporation, Madison, WI, USA) was used for the identification of apoptotic cells, while 4',6-diamidino-2-phenylindole (DAPI; Sigma-Aldrich) staining was applied to identify the normal cells. All the staining experimental protocols were performed by a pathological technician who was blinded to the experiment. Photographs were captured with a fluorescence microscope (Leica Microsystems GmbH, Wetzlar, Germany). A minimum of five fields of view under the microscope were randomly selected for every slice. The number of normal and apoptotic cells were analyzed by Image J $1.44 \mathrm{p}$ software, and the AI was expressed as a percentage of the number of TUNEL-positive nuclei over the total number of nuclei.

Statistical analysis. All data are expressed as the mean \pm standard deviation and were analyzed using one-way analysis of variance. Statistical analysis was performed using SPSS 13.0 software for Windows (SPSS, Inc., Chicago, IL, USA), where $\mathrm{P}<0.05$ was considered to indicate a statistically significant difference.

\section{Results}

HR and MABP measurements. Baseline HR and MABP values were not significantly different among the five groups. Furthermore, the differences were not statistically significant 
Table I. Mean arterial blood pressure $(\mathrm{mmHg})$ of the rats.

\begin{tabular}{|c|c|c|c|c|c|c|}
\hline \multirow[b]{2}{*}{ Group } & \multirow[b]{2}{*}{ Baseline } & \multirow[b]{2}{*}{ Washout } & \multirow[b]{2}{*}{ Ischemia } & \multicolumn{3}{|c|}{ Reperfusion (min) } \\
\hline & & & & 60 & 120 & 180 \\
\hline Sham & $126 \pm 15.7$ & $126 \pm 15.8$ & $121 \pm 17.6$ & $125 \pm 14.2$ & $119 \pm 16.9$ & $120 \pm 24.3$ \\
\hline Control & $126 \pm 14.5$ & $116 \pm 12.1$ & $113 \pm 18.0$ & $114 \pm 13.2$ & $104 \pm 16.8$ & $99 \pm 17.6$ \\
\hline RIPC + RPCT & $134 \pm 12.1$ & $133 \pm 14.9$ & $115 \pm 16.2$ & $114 \pm 11.9$ & $106 \pm 9.8$ & $105 \pm 10.8$ \\
\hline RPCT & $136 \pm 11.8$ & $123 \pm 11.7$ & $123 \pm 11.7$ & $114 \pm 11.1$ & $107 \pm 14.8$ & $101 \pm 19.1$ \\
\hline RIPC & $136 \pm 17.5$ & $124 \pm 18.7$ & $112 \pm 20.6$ & $115 \pm 14.4$ & $113 \pm 20.6$ & $110 \pm 12.7$ \\
\hline
\end{tabular}

Mean arterial blood pressure was measured at the beginning of the experiments (baseline), prior to (washout) and following (ischemia) myocardial ischemia, and after 60, 120 and 180 min of reperfusion. RIPC, remote ischemia preconditioning; RPCT, remote preconditioning of trauma.

Table II. Heart rate (bpm) of the rats.

\begin{tabular}{|c|c|c|c|c|c|c|}
\hline \multirow[b]{2}{*}{ Group } & \multirow[b]{2}{*}{ Baseline } & \multirow[b]{2}{*}{ Washout } & \multirow[b]{2}{*}{ Ischemia } & \multicolumn{3}{|c|}{ Reperfusion (min) } \\
\hline & & & & 60 & 120 & 180 \\
\hline Sham & $314 \pm 12.6$ & $315 \pm 11.4$ & $345 \pm 13.6$ & $325 \pm 10.5$ & $375 \pm 10.2$ & $365 \pm 16.1$ \\
\hline Control & $325 \pm 15.2$ & $328 \pm 14.2$ & $478 \pm 21.4$ & $465 \pm 13.4$ & $475 \pm 13.8$ & $465 \pm 14.7$ \\
\hline RIPC + RPCT & $355 \pm 16.1$ & $375 \pm 8.9$ & $425 \pm 14.2$ & $375 \pm 11.8$ & $396 \pm 12.1$ & $425 \pm 12.4$ \\
\hline RPCT & $333 \pm 14.7$ & $356 \pm 11.4$ & $455 \pm 16.7$ & $396 \pm 12.5$ & $424 \pm 15.4$ & $436 \pm 16.8$ \\
\hline RIPC & $328 \pm 13.3$ & $395 \pm 10.8$ & $475 \pm 18.3$ & $425 \pm 14.6$ & $465 \pm 13.7$ & $453 \pm 15.3$ \\
\hline
\end{tabular}

Heart rates of the rats were measured at the beginning of the experiments (baseline), prior to (washout) and following (ischemia) myocardial ischemia, and after 60, 120 and 180 min of reperfusion. RIPC, remote ischemia preconditioning; RPCT, remote preconditioning of trauma.

\begin{tabular}{|c|c|c|c|c|c|}
\hline & $30 \mathrm{~min}$ & $30 \mathrm{~min}$ & $30 \mathrm{~min}$ & $30 \mathrm{~min}$ & $180 \mathrm{~min}$ \\
\hline sham & baseline & & & & \\
\hline con & baseline & & & occlusion & reperfusion \\
\hline RIPC+RPCT & baseline & RIPC+RPCT & washout & occlusion & reperfusion \\
\hline RPCT & baseline & RPCT & washout & occlusion & reperfusion \\
\hline RIPC & baseline & RIPC & washout & occlusion & reperfusion \\
\hline
\end{tabular}

Figure 1. Experimental protocols. Occlusion and reperfusion of the left anterior descending branch of the left coronary artery were performed in the control, RIPC + RPCT, RPCT and RIPC groups ( $\mathrm{n}=14$ per group). RIPC, remote ischemia preconditioning. RPCT, remote preconditioning of trauma.

among the experimental groups at any time point during the experiments (Tables I and II).

Serum cTnI levels are lower in the experimental groups. Serum levels of cTnI can be used as an indicator of myocardial damage. The three experimental groups exhibited significantly lower serum cTnI levels when compared with the control group at the end of the experiment (control, 58.59 $\pm 12.50 \mathrm{pg} / \mathrm{ml}$; RIPC + RPCT, 46.05 $\pm 8.62 \mathrm{pg} / \mathrm{ml}$; RPCT, 45.98 $\pm 11.24 \mathrm{pg} / \mathrm{ml}$; RIPC, 43.46 \pm 5.05 pg/ml; P<0.05; Fig. 2). However, no statistically significant difference was observed in the serum cTnI level among the three experimental groups. 


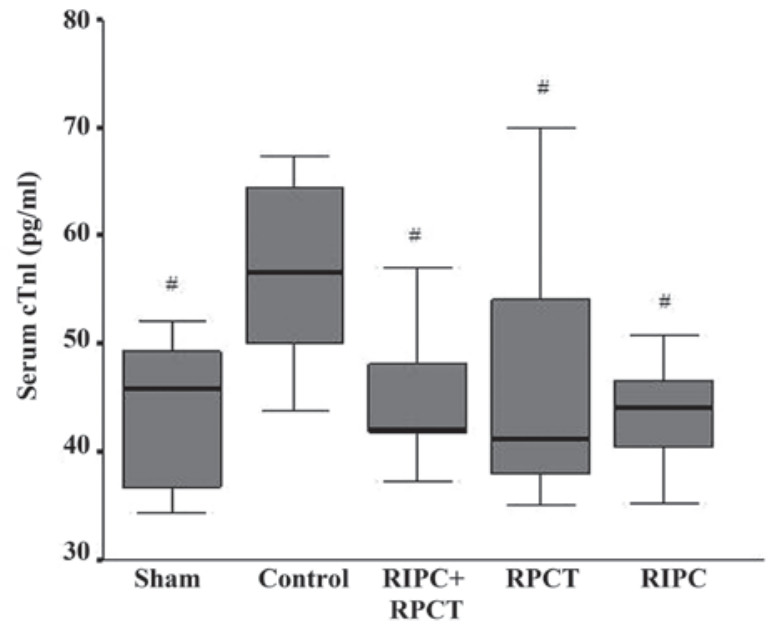

Figure 2. Serum cTnI levels were lower in the experimental groups ( $\mathrm{n}=11$ per group) after $30 \mathrm{~min}$ myocardial ischemia and $180 \mathrm{~min}$ reperfusion. Data are expressed as the mean \pm standard deviation. ${ }^{\#} \mathrm{P}<0.05$, vs. control group. cTnI, cardiac troponin I; RIPC, remote ischemia preconditioning; RPCT, remote preconditioning of trauma.

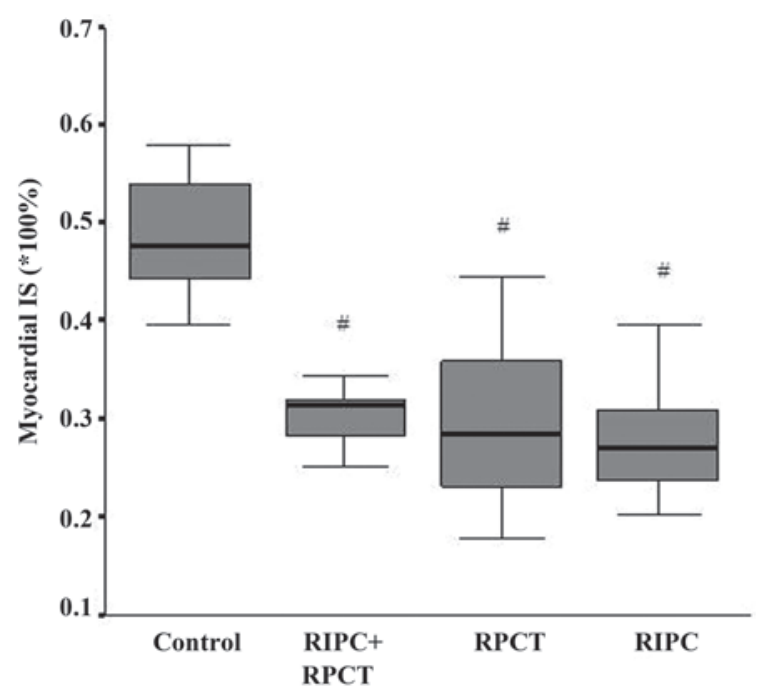

Figure 3. Myocardial IS was reduced in the experimental groups when compared with the control group after 30 min myocardial ischemia and $180 \mathrm{~min}$ reperfusion. No myocardial infarction occured in the Sham group. Data are expressed as the mean \pm standard deviation ( $\mathrm{n}=9$ per group). ${ }^{\#} \mathrm{P}<0.05$, vs. control group. IS, infarct size; RIPC, remote ischemia preconditioning; RPCT, remote preconditioning of trauma.

Preconditioning reduces the myocardial IS. Myocardial IS was expressed as a percentage of the infarct area out of the AAR. In the control group, the IS was $48.34 \pm 6.79 \%$. By contrast, the IS was $29.64 \pm 4.51 \%$ in the RIPC + RPCT group, $29.05 \pm 8.51 \%$ in the RPCT group and $27.72 \pm 6.27 \%$ in the RIPC group. Statistically significant differences were observed when comparing the control group with the experimental groups. $(\mathrm{P}<0.001$; Fig. 3). However, there were no statistically significant differences when comparing the RIPC + RPCT group with the RIPC and RPCT groups.

Fewer apoptotic cardiomyocytes are observed in the experimental groups. The AI was expressed as a percentage of the TUNEL-positive apoptotic nuclei out of the total number of

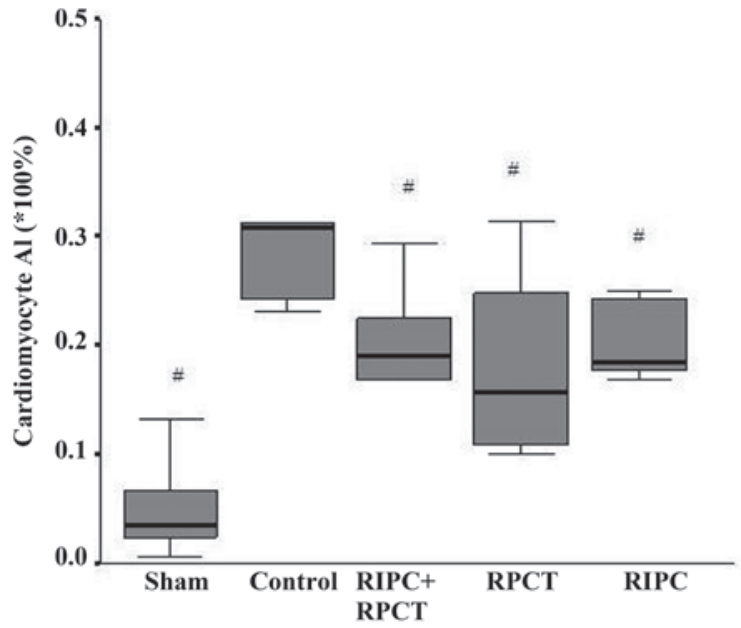

Figure 4. Fewer apoptotic cardiomyocytes were observed in the experimental groups when compared with the control group. The boxplots shows the AI of the cardiomyocytes after 30 min myocardial ischemia and $180 \mathrm{~min}$ reperfusion. Data are expressed as the mean \pm standard deviation ( $\mathrm{n}=5$ per group). ${ }^{\#} \mathrm{P}<0.05$, vs. control group. AI, apoptotic index; RIPC, remote ischemia preconditioning; RPCT, remote preconditioning of trauma.

nuclei. The AI in the control group was determined to be $31.75 \pm 10.65 \%$, and statistically significant differences were observed when compared with the RIPC + RPCT group $(18.32 \pm 9.30 \% ; \mathrm{P}=0.016)$, the RPCT group $(18.51 \pm 9.26 \%$; $\mathrm{P}=0.017)$ and the RIPC group $(20.41 \pm 3.86 \% ; \mathrm{P}=0.038)$. However, no statistically significant differences were identified among the three experimental groups (Figs. 4 and 5).

\section{Discussion}

The results of the present study indicated that RIPC and RPCT exert cardioprotective effects whether used alone or in combination. However, a combination of RIPC with RPCT did not exhibit a stronger cardioprotective effect when compared with either technique applied alone.

A previous study demonstrated that the mechanism underlying RIPC may be mediated by humoral mediators, neurogenic pathways or a combination of the two (4). Following RIPC, the organ releases humoral mediators that are transported to the target organ. These mediators subsequently induce additional humoral mediators at the target organ following transportation, which activate a local neurogenic pathway in the target organ. The activation of afferent nerves in the RIPC organ triggers the release of mediators in the target organ. The RIPC stimulus may also activate a neurogenic pathway in the target organ. A number of mediators may be involved in this mechanism, including adenosine, bradykinin, heat shock proteins, tumor necrosis factor- $\alpha$, nitric oxide and opioids (8-10). However, the mechanism underlying RPCT may depend on the neurogenic pathway. Jones et al (7) proposed that nociceptive stimulation of the peripheral sensory nerve activates cardiac sensory nerves via the spinal nerves at the T9-10 vertebral level, causing cardiac sympathetic nerves to release norepinephrine and bradykinin, which underlies the cardioprotective function following RPCT. Thus, the mechanisms of RIPC and RPCT differ. Nevertheless, the signaling pathway underlying the cardioprotection of RIPC and RPCT at the heart appears to be similar, with involve- 

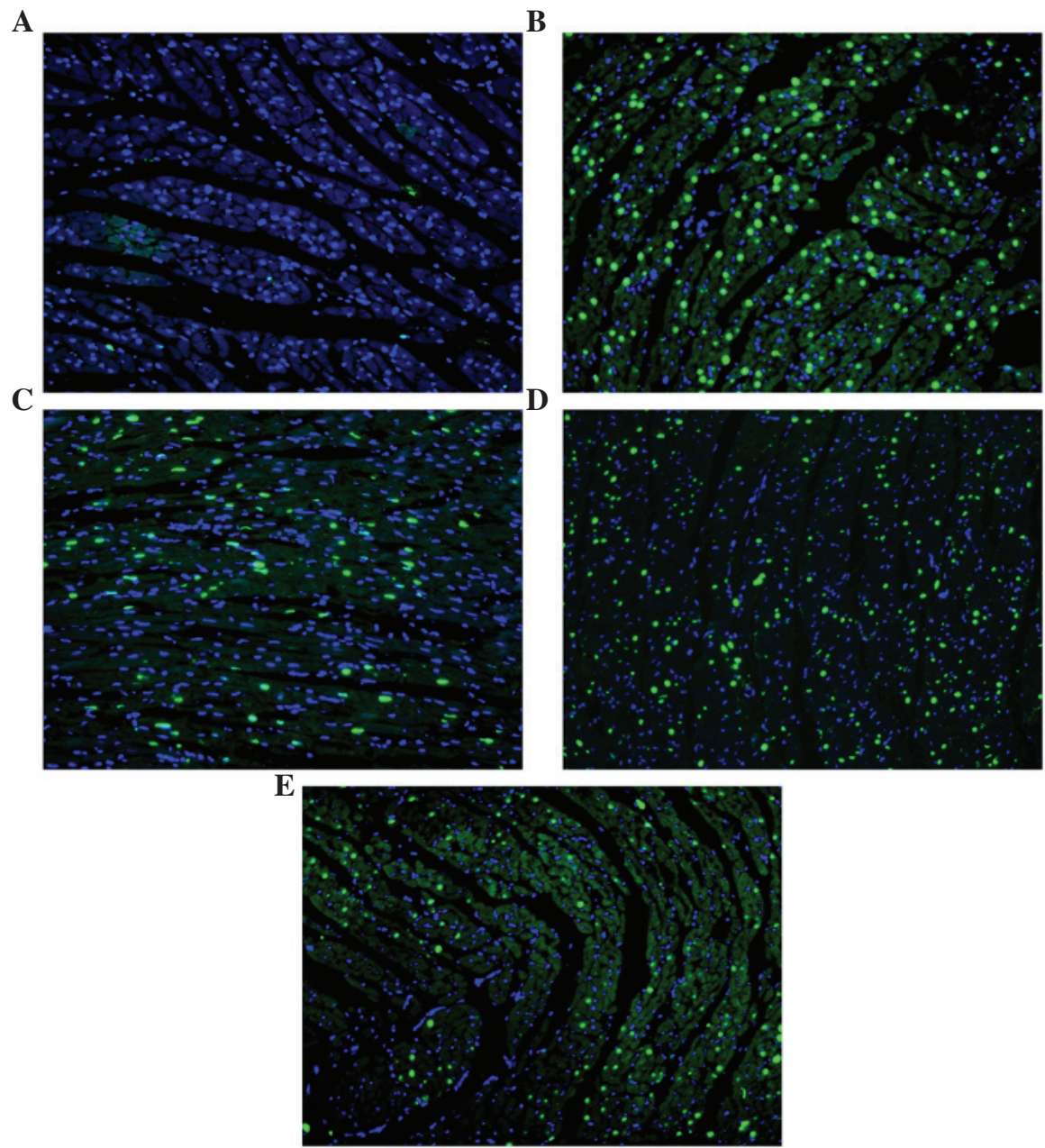

Figure 5. Apoptotic myocytes were stained green using the terminal deoxynucleotidyl transferase-mediated dUTP nick-end labeling technique. Normal cells were stained blue with 4',6-diamidino-2-phenylindole. (A) Sham, (B) control, (C) RIPC+RPCT, (D) RPCT and (E) RIPC groups. RIPC, Remote ischemia preconditioning; RPCT, remote preconditioning of trauma.

ment of protein kinase $\mathrm{C}$, mitrogen-activated protein kinases and mitochondrial potassium ATP, amongst others (7,8,11-13). Therefore, whichever method used to transduce the stimulation of preconditioning to the heart, the mechanism appears to be functioning through the same signaling transduction pathways. Thus, it was hypothesized that this may be the reason as to why RIPC and RPCT, or their combination, did not manifest different cardioprotective effects in the present study.

Currently, an increasing number of elderly individuals with cardiovascular complications are undergoing major surgeries. Perioperative myocardial infarction is one of the main causes of morbidity and mortality in these patients. Therefore, improving the tolerance of the heart to IRI is important for improving the clinical outcome.

Based on the results of the present study, it was hypothesized that for patients undergoing major abdominal surgeries, RIPC is unnecessary since the individuals already undergo RPCT via the abdominal incision at the beginning of the surgery. By contrast, for patients undergoing other forms of non-cardiac major surgery, RPCT is unable to be conducted; thus, RIPC may be useful. However, for patients undergoing cardiac surgery, previous studies performed on human subjects have yielded controversial conclusions. In the study by Karuppasamy et al (14), 54 patients underwent elective coronary artery bypass grafting surgery. The levels of cTnI were analyzed following surgery; however, there were no differences between the RIPC group and the control group. Perrault et al (15) concluded that a number of cardioprotective methods, including hypothermia, cardiopulmonary bypass, cold cardioplegia and intermittent cross-clamp fibrillation, are used routinely in human cardiac surgery, which is in contrast to animal surgery. The combined use of these protective methods renders RIPC negligible. Although RIPC appears to be unnecessary for open chest cardiac surgery, the technique may be useful for types of minimally invasive interventional surgery, such as percutaneous transluminal coronary angioplasty.

By contrast, RIPC may be an effective treatment strategy for a number of other diseases. Hoda et al (16) conducted a study to investigate the therapeutic potential of RIPC in a murine embolic stroke model. The authors found that RIPC was effective alone, but also had additive effects in combination with the administration of intravenous tissue-type plasminogen activator following middle cerebral artery occlusion in mice. The RIPC therapeutic method was demonstrated to improve the cerebral blood flow and neurological outcomes, while reducing the IS. According to the results of the present 
study, RIPC may also be effective for use in other thrombolytic therapies for embolism diseases.

In conclusion, RIPC and RPCT exhibit cardioprotective effects; however, a combination of the two methods does not exert a stronger cardioprotective effect compared with the application of one method alone. Therefore, the results indicate that for patients undergoing major abdominal surgery, RIPC is unnecessary. However, for patient undergoing other types of non-cardiac major surgery and minimally invasive interventional surgery, RIPC may be useful. In addition, RIPC may be considered as an effective procedure for the thrombolytic therapy of embolism diseases.

\section{Acknowledgements}

The authors thank Xiaying Peng, Yanfang Chen and Nanfu Luo for their assistance in the study. In addition, the authors thank Chen Fei and Li Li for their assistance in the pathological procedure.

\section{References}

1. Yellon DM. Hausenloy DJ: Myocardial reperfusion injury. N Engl J Med 357: 1121-1135, 2007.

2. Murry CE, Jennings RB and Reimer KA: Preconditioning with ischemia: a delay of lethal cell injury in ischemic myocardium. Circulation 74: 1124-1136, 1986.

3. Przyklenk K, Bauer B, Ovize M, Kloner RA and Whittaker P: Regional ischemic 'preconditioning' protects remote virgin myocardium from subsequent sustained coronary occlusion. Circulation 87: 893-899, 1993.

4. Lim SY, Yellon DM and Hausenloy DJ: The neural and humoral pathways in remote limb ischemic preconditioning. Basic Res Cardiol 105: 651-655, 2010.

5. Kingma JG Jr, Simard D, Voisine P and Rouleau JR: Role of the autonomic nervous system in cardioprotection by remote preconditioning in isoflurane-anaesthetized dogs. Cardiovasc Res 89: 384-391, 2011.
6. Ren X, Wang Y and Jones WK: TNF-alpha is required for late ischemic preconditioning but not for remote preconditioning of trauma. J Surg Res 121: 120-129, 2004.

7. Jones WK, Fan GC, Liao S, et al: Peripheral nociception associated with surgical incision elicits remote nonischemic cardioprotection via neurogenic activation of protein kinase $\mathrm{C}$ signaling. Circulation 120 (Suppl 11): S1-S9, 2009.

8. Xuan YT, Guo Y, Zhu Y, Wang OL, Rokosh G and Bolli R: Endothelial nitric oxide synthase plays an obligatory role in the late phase of ischemic preconditioning by activating the protein kinase $C$ epsilon $\mathrm{p} 44 / 42$ mitogen-activated protein kinase pSer-signal transducers and activators of transcription $1 / 3$ pathway. Circulation 116: 535-544, 2007.

9. Wong GT, Ling Ling J and Irwin MG: Activation of central opioid receptors induces cardioprotection against ischemia-reperfusion injury. Anesth Analg 111: 24-28, 2010.

10. Kanoria S, Jalan R, Seifalian AM, Williams R and Davidson BR: Protocols and mechanisms for remote ischemic preconditioning: a novel method for reducing ischemia reperfusion injury. Transplantation 84: 445-458, 2007.

11. Sadat U: Signaling pathways of cardioprotective ischemic preconditioning. Int J Surg 7: 490-498, 2009.

12. Das B and Sarkar C: Is preconditioning by oxytocin administration mediated by iNOS and/or mitochondrial K(ATP) channel activation in the in vivo anesthetized rabbit heart? Life Sci 90: 763-769, 2012.

13. Gross GJ, Baker JE, Moore J, Falck JR and Nithipatikom K: Abdominal surgical incision induces remote preconditioning of trauma (RPCT) via activation of bradykinin receptors (BK2R) and the cytochrome P450 epoxygenase pathway in canine hearts. Cardiovasc Drugs Ther 25: 517-522, 2011.

14. Karuppasamy P, Chaubey S, Dew T, et al: Remote intermittent ischemia before coronary artery bypass graft surgery: a strategy to reduce injury and inflammation? Basic Res Cardiol 106: 511-519, 2011.

15. Perrault LP, Menasché $\mathrm{P}, \mathrm{Bel} \mathrm{A}$, et al: Ischemic preconditioning in cardiac surgery: a word of caution. J Thorac Cardiovasc Surg 112: 1378-1386, 1996.

16. Hoda MN, Siddiqui S, Herberg S, et al: Remote ischemic perconditioning is effective alone and in combination with intravenous tissue-type plasminogen activator in murine model of embolic stroke. Stroke 43: 2794-2799, 2012. 